\title{
EDDIE - 3D SCANNING AND AUGMENTED REALITY IN EDUCATION
}

\author{
Csaba JUHÁSZ1 ${ }^{1}$, Kristóf MUHI ${ }^{2}$ Zsolt Csaba JOHANYÁK ${ }^{3}$ \\ Neumann János University, GAMF Faculty of Engineering and Computer Science, Kecskemét, Hungary \\ ${ }_{1}$ juhaszcsaba23@gmail.com \\ ${ }^{2}$ mkristof@outlook.com \\ 3johanyak.csaba@gamf.uni-neumann.hu
}

\begin{abstract}
We are convinced that the learning process could be a lot more interesting and more interactive for young people with the help of modern technologies. The presence and the usage of the smartphones is natural for today's young generations, although they do not use their inherent potential for example for educational purposes. Eddie is a hybrid of a smartphone application and a school book. The school book would keep its original form, but we would make the application fit for a given subject. The already existing pictures in the schoolbooks would work as a special indicator, which our application could read. The application would show different contents virtually in AR (Augmented Reality), based on the scanned image. The shown content could be a 3D model, animation or video.
\end{abstract}

Keywords: education, augmented reality, digital education, smartphone, application.

\section{Background}

\subsection{The Augmented Reality}

Augmented Reality (AR) is a sort of a virtual extension of reality. For practical use we need a smartphone with the right hardware and with a camera, or special glasses made for this purpose.

Through these specific devices' camera we are able put different elements into the real environment in real time.

Development wise we can divide Augmented Reality into two main categories. The algorithm which defines the virtual element's place, and the display mode.

One is the position and direction-based AR. Its goal is to add extra information to the camera

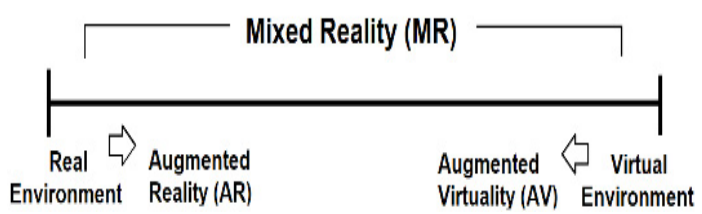

Figure 1. Categories of mixed reality [1] view which is already on the device's screen. This extra information is called the POIs (Point of Interests). These points contain places which can be interesting, useful for the users. The correct information which is displayed on the screen is defined by processing data from the GPS position, the integrated compass and the accelerometer.

The other type of AR is marker-based AR. In this case we are looking for a special indication (usually a picture, or part of a picture). The position of the marker (indicator) is defined by the real time image of the camera and this way we can put a virtual object on top of the picture. Our application falls into this category as well.

\subsubsection{Historical review}

In 1986 Ivan Sutherland [2] fdeveloped the first screen system which was attachable to the head. The system was able to show different wireframe graphics.

In 1974 Myron Krueger built a labyrinth called Videoplace [3] which was able to simulate lifelike silhouette with different projectors and cameras, 
surrounding the users with multiple interactive user interfaces.

After much research and development, the next milestone was in 1990 when Tom Caudell created the expression Augmented Reality (AR) [4]. In 1994 Louis Rosenberg developed the first working AR system for the American Airforce [5] to control different virtual machines. In $1994 \mathrm{Ju}-$ lie Martin organises the first theatre production which involved the new AR technology, where the actors were dancing around a virtual object on the stage.

From the beginning of the next decade AR's field of usage won a huge space in entertainment industry. First in 1998 the Sportvision TV channel used visual indicators in NFL [6] to highlight the different elements in the game.

In the military industry there were consistent development for visual help tools, especially in the air force. Hirokazu Kato invented the ARToolKit [7] which is an open source program library. With the help of it we can show different computer graphics in a real time camera image and we are also able to track them. Since 2009 ARToolKit has supported web browsers as well.

Nowadays with the rapid progression of hardware and software we are getting closer to lifelike AR experiences. All the huge IT companies are focusing on this technology. The Microsoft HoloLens [8] ] glass can show AR content with no additional hardware, without indicators, detecting the space and flat surfaces. Apple and Google, the developers of the two biggest smartphone operating systems, iOS and Android, have their own AR developer environment, and with these environments we are able to create augmented reality experiences.

\subsubsection{Field of usages}

As mentioned in the last section, AR technology was used first in live sport broadcasts. Projecting the soccer team's logos onto the field or projecting the finish line on the water in kayak-canoe competitions uses the same technology as well. It is usable for marketing purposes as well. For example, in tourism the direction-based AR provides wider variety of information about the city around us. And just another few examples: healthcare, industrial processes (manufacturing and repairing), entertainment, education, gaming, etc.

\section{The planning of Eddie application}

The goal of the application is to extend the traditional education system, and add an interactive, experience based tool to help the learning process. The application can visualise, show different digital contents (mostly 3D objects) on the user's smart device (smartphone or tablet) using AR technology.

The visualisation in AR is quite device limited, so choosing the right development environment was a key task. The other key point of the planning was to make the application easy to use for students and teachers, which means they needn't buy extra devices (for example AR glasses), the application should be easy to obtain and install. For teachers the 3D content preparation (creating collections) must be effortless

\subsection{Choosing development environment}

\subsubsection{AR development environments}

The rapid progression of AR technology has resulted in there being no need to have high-end devices to visualise. Because of this it provides the opportunity to provide an increasingly better AR experience for those users with average devices.

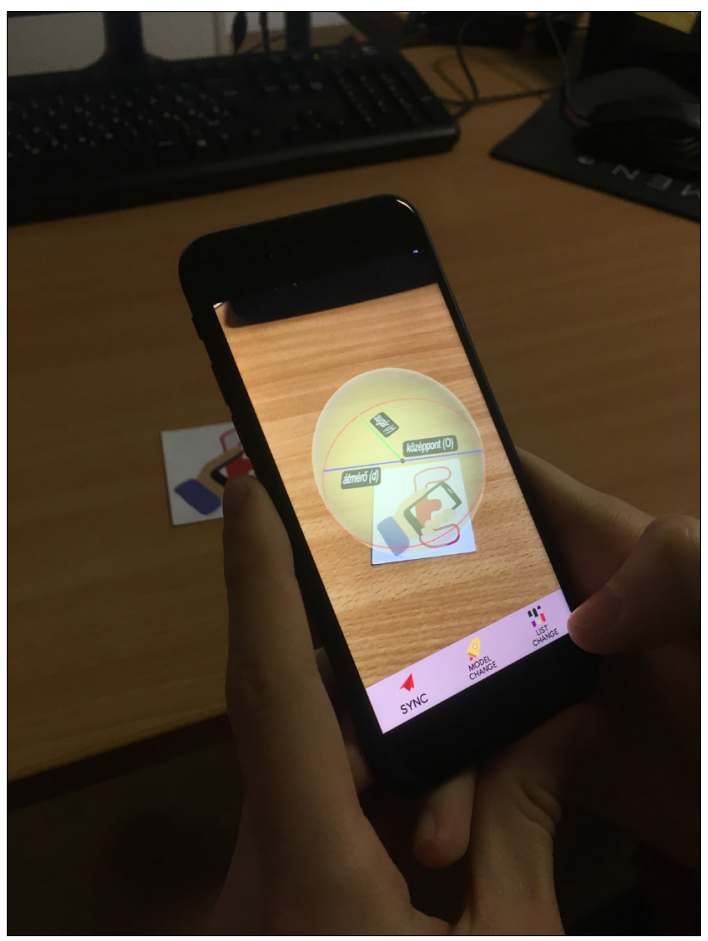

Figure 2. Eddie application in action 
At the planning phase of the application we decided not to limit the devices, we wanted to provide this application for as many users as possible.

In the next section we highlight $3 \mathrm{AR}$ development libraries.

\subsubsection{ARCore}

The ARCore [9] is an AR displaying SDK (software development kit) developed by Google. Its first version was released on 1st of March 2018.

It uses 3 different technologies to show digital content: motion tracking, environmental recognition (flat surfaces, floor recognition) and processing the environmental lights. The usage requires Android 7.0 (Nougat) or later operating system.

On Apple's operating system it requires version 11.0. Next to the OS requirements it has device requirements as well. The ARCore works only on Apple iPhone SE or later devices (15 devices). Of those phones which have the Android system right now only around 100 devices support it from the 21700 different [10] smartphones.

Because of the strong device requirements mentioned above ARCore library was not good enough for our goals and plans.

\subsubsection{ARKit}

The ARKit [11] is an AR displaying library developed by Apple. Its first stable version was published at the same time when iOS 11 was released in June of 2017.

The library works exclusively on iPhones and iPads. There are some software and hardware limitations as well, just like with ARCore: it requires iOS 11 or later and iPhone SE or later devices. Technologically it uses the iPhone's hardware, for example the VIO (Visual Inertial Odometry) which is responsible for recognizing the environment (recognition of flat surfaces, objects, floor etc.). It can recognise and process the light coming from other sources for a better visualisation, just like ARCore.

Just like Google's ARCore's requirements, ARKit's iOS requirements couldn't match with the development's goals and plans.

\subsubsection{Vuforia}

Vuforia (QCAR earlier) is an AR developer platform developed by Qualcomm. Its first stable version was released in April of 2011. [12] It provides a marker-based SDK for AR development. It has better device support than the libraries mentioned earlier, ARKit and ARCore. It does have some software requirements: it requires Android
4.4 or later and iOS 9.0 or later. Vuforia also has a Cloud service which allows us to change the markers and the connected digital, the displayed AR content in real time.

If we look at the requirements from our side, which was mentioned earlier, Vuforia's wide range of device support and its Cloud service made us to stick with this library for the development.

\subsection{D scanning}

3D scanning is used when the want to improve the efficiency for modelling a difficult geometric 3D model, which cannot be built up from easy geometrical elements. When you scan an object, the scanned model behaves as a template and makes the engineering task a lot easier and, we can look at it as a finished, customisable model.

The 3D model resulting from 3D scanning can be texturized or can be a mesh [13]. Scanning a human body takes around 5-15 minutes with the instructions. The actual scanning takes 2-3 minutes. Scanning objects can take more than a few days if the object needs to be remodelled.

The goal of 3D scanning is to get a point-cloud which can help us to create a model, to create segments, to create precise documentation, for reconstruction, for measuring, to prepare the object for 3D printing and to calculate the object's volume.

Most popular field of usages:

- architecture;

-industry (car-, machine-, tool manufacturing);

- reverse engineering

We followed through the process of reverse engineering while we were scanning our objects (biological models).

\subsection{Reverse engineering}

Reverse engineering is a kind of process, where we create a blueprint for a finished object which does not have a blueprint, a digitalised draft plan, a handmade master sample, a complex object, or a fitting part.

The process of reverse engineering:

- digitalising the physical object;

- adjusting the measuring points;

-importing the measuring points into a CAD system;

- fitting surface curves;

- surface or surface plane alignment on curves;

-removing the useless parts;

-fixing the model;

- manufacturing the model;

-measurement 


\section{Findings}

AR technology and the pros/cons given by 3D scanning technology: While researching and experimenting with the 3D scanner, Sense3D [14] we realised that its low quality scanning ability is not enough to use it universally. Because choosing the right AR content displaying library, Vuforia, we can have significant performance advantage even if AR technology has its software and hardware limits.

To improve the application and to continue developing it properly, most importantly, we need the customers' feedback. Because of this we would like to organise workshops and meetings where the students and the teachers can try out the application interactively and we could collect valid feedback.

Improving the quality of the 3D scanning is essential from the viewpoint of the right experience.

\section{Acknowledgement}

This research is supported by EFOP-3.6.1-16-201600006 "The development and enhancement of the research potential at John von Neumann University" project. The Project is supported by the Hungarian Government and co-financed by the European Social Fund.

\section{References}

[1] Milgram P., Takemura H., Utsumi A.: Augmented Reality: A class of displays on the reality-virtuality continuum. Proceedings of Telemanipulator and Telepresence Technologies, 1994

[2] Colocation America, The history of augmented reality. (2018-11-04) https://www.colocationamerica.com/blog/history-of-augmented-reality

[3] The Digital Age, Myron Krueger Biography http://thedigitalage.pbworks.com/w/ page/22039083/Myron\%20Krueger (2018-11-04)

[4] AR at Boeing (1990) (2018-11-04) http://www.idemployee.id.tue.nl/g.w.m.rauterberg/presentations/hci-history/tsld096.htm

[5] Infographic, The History of Augmented Reality http://www.augment.com/blog/infographic-lengthy-history-augmented-reality/ (2018-11-04)

[6] Sports Illustrated, Behind the NFL's yellow first down line, and what's next for sports TV

https://www.si.com/edge/2015/01/29/behind-nfl-yellow-first-down-line-sportsvision-technology (2018-11-04)

[7] Apple Developer, ARToolKit (2018-11-04) http://www.hitl.washington.edu/artoolkit/

[8] Microsoft, HoloLens. (2018-11-04) https://www.microsoft.com/hu-hu/hololens

[9] Google, ARCore. (2018-11-04) https://developers.google.com/ar/

[10] Android supported devices list. (2018-11-04) http://storage.googleapis.com/play_public/supported_devices.csv

[11] Apple Developer, ARKit. (2018-11-04) https://developer.apple.com/arkit/

[12] Vuforia AR Engine. (2018-11-04) https://www.vuforia.com/

[13] Freedee, A non-kontakt 3D szkennerek helye a gyártásban

http://www.freedee.hu/a-non-kontakt-3d-szkennerek-helye-a-gyartasban/ (2018-11-04)

[14] iDig, Sense 2 3D scanner. (2018-11-04) https://www.idig3dprinting.co.uk/shop/brand/ sense-3d-scanner 